\title{
OPTIMUM DESIGN OF SMALL-SIGNAL MICROWAVE AMPLIFIERS WITH SPECIFIED STABILITY SAFETY MARGIN
}

\author{
K. W. ECCLESTON \\ Dept of Electrical Engineering \\ National University of Singapore \\ SINGAPORE 119260 \\ E-mail: elekwe@nus.edu.sg
}

\begin{abstract}
When employing conditionally stable transistors in microwave amplifier design, it is impossible to have both ports perfectly matched. One can easily design for a perfect match at one of the ports, but the resulting mismatch at the remaining port may be unacceptable. Through trial and error using graphical methods, the designer may trade-off the perfect port match for improved match at the other port. In this paper, we propose a systematic approach whereby the maximisation of an objective function results in maximum gain and port match, for given stability safety margins.
\end{abstract}

\section{Introduction}

When designing microwave amplifiers, it is the task to select suitable values for the source (input generator) and load reflection coefficients for the transistor. The source and load reflection coefficients are chosen to achieve minimum gain and port matching, as well as ensure stability. When using conditionally stable transistors, it is impossible to simultaneously achieve perfect match at both ports. However, one can easily design an amplifier with one of the ports perfectly matched, but the resulting mismatch at the other port is often unacceptable. Therefore it is often necessary to trade-off the perfect port match for improved match at the other port.

Amplifier design typically begins with the selection of either the source or load reflection coefficient so that a certain gain can be achieved as well as allowing conjugate matching at the other port [1]. The appropriate gain circles, along with the appropriate stability circle, are plotted on either the source or load reflection coefficient plane to aid selection of the first reflection coefficient [2]. An appropriate safety margin between the chosen value of the first reflection coefficient and stability circle is necessary to ensure conjugate matching can be used at the other port [1], but also allows for manufacturing variations [3]. One can further ensure adequate stability safety margin at the remaining port [3]. Although the resulting amplifier is matched at one of its ports, the mismatch at the other port is typically severe and necessitates the trade-off perfect match at one port for improved match at the other. Circles of constant mismatch or VSWR [2] can be described on the reflection coefficient planes to aid this trade-off.

The attractiveness of these graphical design methods is that the functional behaviour of gain, and mismatch with respect to the source and load reflection coefficients is obtained by simply plotting circles whose centres and radii need only be calculated using well known formulae [2]. A disadvantage of this approach is that the selection of the source and load reflection coefficients is subjective, particularly when the contours of more than two performance parameters are plotted on the same reflection coefficient plane. "Trial and error" is often required to obtain acceptable performance, and inevitably, circuit optimisers are required.

Recent research activities in microwave amplifier design have focussed on stability [1][3][4] or noise performance [5] - [7]; but apart from Albinsson [6], do not addressed mismatch. In previous work by the author [8], the design of a low noise microwave amplifier was considered. To optimise the LNA, two objective functions were considered: one being the noise measure, and the other being the product of the two port mismatches. The former was first minimised with respect to the source stability safety margin and intermediate variable, whilst the later was maximised with respect to the load stability safety margin and another intermediate variable. By adopting this approach, the stability 
safety margin is inherently incorporated into the design since it is a design variable [8]. However, a problem with this approach is that being an LNA design, it was necessary to consider two objective functions and hence optimisation in two steps.

In this paper, we propose a design approach for a microwave amplifier gain stage whereby it is possible to incorporate gain and port mismatch into one objective function meaning that the amplifier can be optimised in one step. In a similar approach to the previous work [8], this objective function can be related to the source and load stability safety margins, as well as intermediate variables, rather than directly to the source and load reflection coefficients. For given stability safety margins, the objective function is easily maximised with respect to two intermediate variables using a simple direct search method.

\section{Definitions}

In the work that follows, the definitions of the various reflection coefficients refer to the single-stage amplifier depicted in Fig. 1. The transistor is described by its S-parameters, the effective source and load reflection coefficients presented to the input and output ports of the transistor are $\Gamma_{\mathrm{S}}$ and $\Gamma_{\mathrm{L}}$ respectively. In this work, we will assume that the magnitudes of the transistor S-parameters $S_{11}$ and $S_{22}$ are both less than unity and that the stability circles do not enclose the origin. These assumptions are valid for many microwave transistors used in amplifier circuits. $\Gamma_{1}$ is the reflection coefficient looking into the input port of the transistor and is a function of $\Gamma_{\mathrm{L}}$ via the well known bilinear transformation [2]. Similarly $\Gamma_{2}$ is the reflection coefficient looking into the output port of the transistor and is a function of $\Gamma_{\mathrm{S}}$ via the well known bilinear transformation [2].

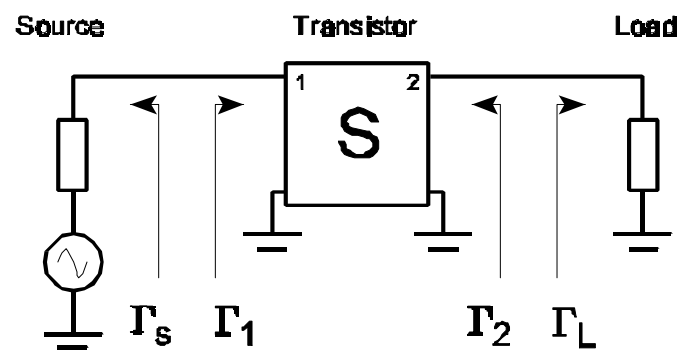

Figure 1. Microwave amplifier circuit.

In general, $\Gamma_{\mathrm{S}}$ and $\Gamma_{1}$ are mismatched with mismatch factor $M_{1}$ :

$$
M_{1}=\frac{\left(1-\left|\Gamma_{S}\right|^{2}\right)\left(1-\left|\Gamma_{1}\right|^{2}\right)}{\left|1-\Gamma_{S} \Gamma_{1}\right|^{2}}
$$

similarly, $\Gamma_{\mathrm{L}}$ and $\Gamma_{2}$ are mismatched with mismatch factor $M_{2}$ :

$$
M_{2}=\frac{\left(1-\left|\Gamma_{L}\right|^{2}\right)\left(1-\left|\Gamma_{2}\right|^{2}\right)}{\left|1-\Gamma_{L} \Gamma_{2}\right|^{2}}
$$

In the presence of statistical variation of both transistor and circuit parameters, it is important when selecting $\Gamma_{\mathrm{S}}$ and $\Gamma_{\mathrm{L}}$ that an adequate safety margin with respect to their respective stability circles be maintained. Eccleston [3] has previously developed equations for maximum gain design using conditionally stable transistors and defined a new performance parameter, the stability safety margin. Let $C_{S}$ and $r_{S}$ be the centre and radius of the source stability circle, and $C_{L}$ and $r_{L}$ be the centre and radius of the load stability circle. The source safety margin $(S S M)$ is the distance between the nominal (and allowable) value of $\Gamma_{\mathrm{S}}$ and the source stability circle. Likewise, the load safety margin $(L S M)$ is the distance between the nominal (and allowable) value of $\Gamma_{\mathrm{L}}$ and the load stability circle. 


\section{Objective Function Formulation}

In the approach that we propose, the functional behaviour of noise measure and port mismatch are described as functions of the stability safety margins rather than direct functions of $\Gamma_{\mathrm{S}}$ and $\Gamma_{\mathrm{L}}$. Since we assume that the transistor S-parameters $S_{11}$ and $S_{22}$ are both less than unity and that the stability circles do not enclose the origin, we can write $\Gamma_{S}$ and $\Gamma_{\mathrm{L}}$ in terms of stability circle centres and radii, $S S M$ and $L S M$ :

$$
\begin{aligned}
& \Gamma_{\mathrm{S}}=C_{S}+\left(r_{S}+S S M\right) \exp (j \theta) \\
& \Gamma_{\mathrm{L}}=C_{L}+\left(r_{L}+L S M\right) \exp (j \phi)
\end{aligned}
$$

where $\theta$ and $\phi$ are intermediate design parameters. With $\Gamma_{\mathrm{S}}$ and $\Gamma_{\mathrm{L}}$ described in this manner, both gain and mismatch factor can be described as functions of SSM, LSM, $\theta$ and $\phi$. Thus SSM and LSM are design parameters as well as performance parameters.

The objective functions we wish to develop consider both gain and mismatch at both ports. Therefore it is possible to define two such objective functions:

$$
\text { and } \quad \begin{aligned}
& \mathrm{F}_{\mathrm{A}}(\theta, \phi, \mathrm{SSM}, \mathrm{LSM})=\mathrm{M}_{1} \mathrm{G}_{\mathrm{A}} \mathrm{M}_{2} \\
& \mathrm{~F}_{\mathrm{P}}(\theta, \phi, \mathrm{SSM}, \mathrm{LSM})=\mathrm{M}_{1} \mathrm{G}_{\mathrm{P}} \mathrm{M}_{2}
\end{aligned}
$$

where $G_{A}$ and $G_{P}$ are the available gain and power gain respectively. Because the products $G_{A} M_{2}$ and $M_{1} G_{P}$ give the transducer gain, it is not necessary to consider another objective function involving the transducer gain. We see that maximising either objective function with respect to $\theta$ and $\phi$ for given stability safety margins (SSM and LSM) will simultaneously yield high gain and good port match. The choices available is between application of either (5) or (6) and the appropriate values of SSM and LSM. The appropriate choice of SSM and LSM is related to expected manufacturing process variations. The search domain for $\theta$ and $\phi$ may be determined from (3) and (4) by noting that the resulting values of $\Gamma_{S}$ and $\Gamma_{L}$ must have magnitudes less than unity. With optimum values of $\theta$ and $\phi$ determined, the corresponding optimum values of $\Gamma_{S}$ and $\Gamma_{L}$ may be obtained using (3) and (4).

\section{Numerical Examples}

To illustrate the application of the above objective functions (5) and (6), we can consider a transistor whose S-parameters are:

$$
\begin{array}{ll}
S_{11}=0.809 \underline{/-148^{\circ}} & S_{12}=0.111 \underline{/-4^{\circ}} \\
S_{21}=1.940 / 57^{\circ} & S_{22}=0.634 \underline{/-48^{\circ}}
\end{array}
$$

Figure 2 shows the contour plot of $\mathrm{F}_{\mathrm{A}}$ and $\mathrm{F}_{\mathrm{P}}$ as functions of $\theta$ and $\phi$ (for complete search domain) for SSM and LSM both equal to 0.1. We see from Figure 2 that the local maximum is well defined for both objective functions and can be easily located by direct search of the discretised $\theta \phi$ plane. For example, $\mathrm{F}_{\mathrm{A}}$ is maximised at $\theta=-0.3719, \phi=4.81$, and hence application of (3) and (4) yields the corresponding optimum values for reflection coefficients: $\Gamma_{\mathrm{S}}=0.804 / 171^{\circ}$ and $\Gamma_{\mathrm{L}}=0.812 / 62^{\circ}$. Table I shows the maximum objective function value, and corresponding gain and mismatch, for various values of SSM and LSM. The maximum of $F_{A}$ or $F_{P}$ was located by sampling the search domain at the grid points of a 100 by 100 grid discretisation of the $\theta \phi$ plane. We see from Table $\mathrm{I}$, that the outcome when either using $\mathrm{F}_{\mathrm{A}}$ or $\mathrm{F}_{\mathrm{P}}$ are in both cases good but using $\mathrm{F}_{\mathrm{P}}$ results in about $0.7 \mathrm{~dB}$ improvement over using $\mathrm{F}_{\mathrm{A}}$ in this case.

\section{Conclusion}

In this paper we have shown a method for selecting the values of the source and load reflection coefficients to achieve maximum gain and match at both ports, for given stability safety margins. 
This method is based on an objective function that is described in terms of stability safety margins, thereby incorporating stability into the design parameters rather than consider them separately. The objective functions are straightforward to maximise without sophisticated optimisers.

\section{References}

[1] M. L. Edwards, S. Cheng \& J. H Sinsky, "A Deterministic Approach for Designing Conditionally Stable Amplifiers", IEEE T. on MTT, 41(2), July 1995, pp 1567 - 1575.

[2] G. Gonzalez, Microwave Transistor Amplifiers, Analysis and Design, Prentice-Hall 1997.

[3] K. W. Eccleston, "Design Formulae for Microwave Amplifiers Employing Conditionally-Stable FETs", IEICE Trans. on Electronics, E82-C(7), July 1999, pp 1054 - 1060.

[4] M. L. Edwards \& S. Cheng, "Conditionally Stable Amplifier Design Using Constant $\mu$ - Contours", IEEE T. on MTT, 44(12), Dec. 1996, pp 2634 - 2640.

[5] C. R. Poole \& D. K. Paul, "Optimum Noise Measure Terminations for Microwave Transistor Amplifiers", IEEE T. on MTT, 33(11), November 1985, pp 1254 -1257.

[6] B. M. Albinsson, "A Graphic Design Method for Matched Low-Noise Amplifiers, IEEE T. on MTT, 38(2), February 1990, pp 118-122.

[7] G. N. Link \& V. S. Gudimetla, "Analytical Expressions for Simplifying the Design of Broadband Low Noise Microwave Amplifiers", IEEE T. on MTT, 43(10), October 1995, pp 2498 - 2501.

[8] KW Eccleston, "Stability Safety Margin Based Design of Low Noise Microwave Amplifiers", APMC '99, 30 Nov - 3 Dec 1999, Singapore, pp 1 - 4.

Table I Optimum Gain and Mismatch Factors

\begin{tabular}{|c|c|c|c|c|c|c|c|c|}
\hline $\begin{array}{c}\text { SSM / } \\
\text { LSM }\end{array}$ & \multicolumn{4}{|c|}{ Maximising objective function $\mathrm{F}_{\mathrm{A}}$} & \multicolumn{3}{c|}{ Maximising objective function $\mathrm{F}_{\mathrm{P}}$} \\
\cline { 2 - 9 } & Max $\mathrm{F}_{\mathrm{A}}$ & $\begin{array}{c}\mathrm{G}_{\mathrm{T}} \\
(\mathrm{dB})\end{array}$ & $\mathrm{M}_{1}$ & $\mathrm{M}_{2}$ & Max $\mathrm{F}_{\mathrm{P}}$ & $\begin{array}{c}\mathrm{G}_{\mathrm{T}} \\
(\mathrm{dB})\end{array}$ & $\mathrm{M}_{1}$ & $\mathrm{M}_{2}$ \\
\hline 0.1 & 19.9 & 13.9 & 0.818 & 0.835 & 24.6 & 14.2 & 0.587 & 0.933 \\
\hline 0.2 & 15.4 & 12.5 & 0.859 & 0.898 & 18.4 & 12.8 & 0.708 & 0.972 \\
\hline 0.3 & 12.4 & 11.6 & 0.864 & 0.903 & 14.6 & 11.8 & 0.734 & 0.974 \\
\hline 0.4 & 10.0 & 10.7 & 0.851 & 0.889 & 11.8 & 10.9 & 0.730 & 0.964 \\
\hline
\end{tabular}
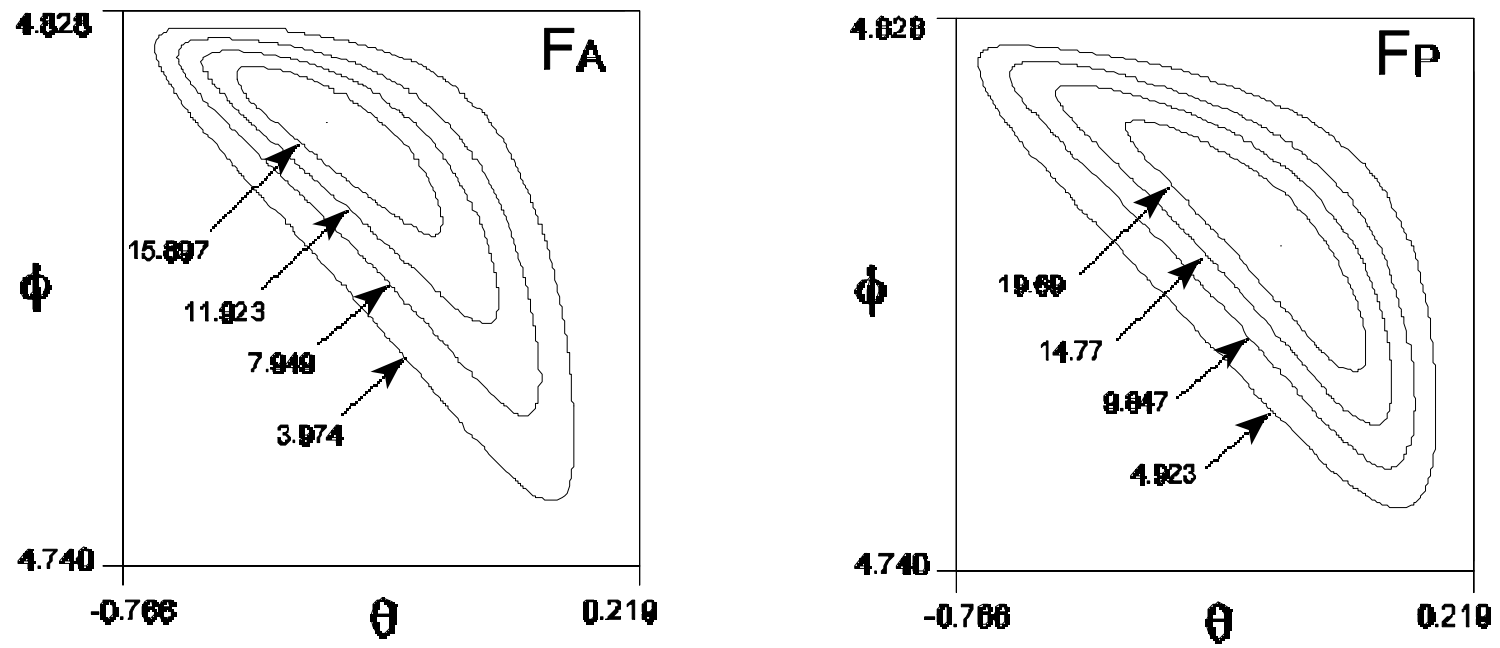

Figure 2. Contour plots of objective functions $\mathrm{F}_{\mathrm{A}}$ (left) and $\mathrm{F}_{\mathrm{P}}$ (right) for SSM and LSM equal to 0.1. 